\title{
Competencias lectoras y escritoras a través de la investigación como estrategia pedagógica ${ }^{1}$
}

\section{$\overline{\text { Reading competencies and writers through }}$ the research as a pedagogical strategy}

DOI: http://dx.doi.org/10.17981/cultedusoc.9.3.2018.0

\author{
Artículo de investigación. Fecha de recepción: 15/06/2018. Fecha de aceptación: 27/11/2018 \\ Odalis Vásquez-Bustamante ${ }^{2}$ \\ Institución Educativa Fossy Marcos María, sede principal (Colombia) \\ oldalisbus@hotmai.com
}

Para citar este artículo:

Vásquez-Bustamante, O. (2018). Competencias lectoras y escritoras a través de la investigación como estrategia pedagógica. Cultura. Educación y Sociedad 9(3), 9-18. DOI: http://dx.doi.org/10.17981/cultedusoc.9.3.2018.0

\section{Resumen}

Las competencias lectoras son integradas dentro del desarrollo de cualquier relación social fomentando la participación, lo cual implica diversas variables y niveles del lenguaje oral. El estudio buscó fortalecer las competencias lectoras y escritoras a través de la IEP en la educación básica primaria. Se abordó bajo los lineamientos metodológicos de la IEP, con un diseño basado en las trayectorias de indagación, utilizando técnicas propias de la investigación cualitativa necesaria para la recolección de información. Como unidad de análisis se seleccionaron cuarenta y dos (42) estudiantes de tercero a quinto de las sedes Delicias y principal de la Institución Educativa Departamental Fossy Marcos María, sede principal. Se concluye que la muestra seleccionada tiene deficiencias en los procesos de lectura y escritura. Situación que motivó a desarrollar el presente estudio para fortalecer competencias lectoras con el objetivo de mejorar la fluidez, comprensión lectora y producción oral, y escrita.

Palabras clave: Competencias lectoras, competencias escritoras, investigación como estrategia pedagógica.

\section{Abstract}

Reading skills are integrated into the development of any social relationship by encouraging participation, which involves various variables and levels of oral language. The study sought to strengthen reading and writing skills through the IEP in primary basic education. It was addressed under the methodological guidelines of the IEP, with a design based on the trajectories of inquiry, using techniques of qualitative research necessary for the collection of information. As a population under study, forty-two (42) students from third to fifth were selected from the Delicias and main offices of the Departmental Educational Institution Fossy Marcos María, headquarters. It is concluded that the selected sample has deficiencies in the reading and writing processes. Situation that motivated to develop the present study to strengthen reading skills with the aim of improving fluency, reading comprehension and oral and written production.

Keywords: Reading competencies, skills writers, research and pedagogical strategy.

1 Este artículo ha sido derivado del Programa de Fortalecimiento de la Cultura Ciudadana y Democrática CT+I a través de la IEP apoyada en TIC en el Departamento de Magdalena: CICLON

2 Docente de la institución educativa Fossy Marcos María, sede principal, del grupo de investigación Mariposas amarillas.

- The author; licensee Universidad de la Costa - CUC.

Cultura, Educación y Sociedad vol. 9 no. 3, pp. 9-18. Diciembre, 2018

Barranquilla. ISSN 2389-7724 Online 


\section{Introducción}

En los últimos años el ambiente educativo se ha visto enriquecido por aportes teóricos de diversas disciplinas y la tecnología de información y comunicación (TIC), donde las investigaciones en el área del lenguaje en el primer ciclo de básica primaria se han limitado al estudio del proceso de aprendizaje de la lectura y la escritura, mientras que son pocas las que abordan el rol del docente como mediador y facilitador del desarrollo de competencias lecto- escritoras.

Para la lectura, la informatización tiene consecuencias aparentemente contradictorias, ser lector es ahora, según se mire, más fácil o difícil. La información es mucho más abundante e inmediata, y los canales de producción y acceso cada vez menos selectivos. Por otra parte, interactuar con el flujo incesante de información en la que con frecuencia confluyen elementos distractores de enorme atractivo y que favorecen una mente de malabarista, exige competencias que no requiere en el mismo grado la lectura de información analítica de textos ordenados y concebidos según una lógica, (Carr, 2011).

De hecho, todo esto posibilita acercarse al lector actualizado, que procesa el texto, que accede al conocimiento de otros (e incrementa y transforma el suyo propio) a través de la lectura de múltiples textos, que son leídos por y para uno mismo, en un silencio conducente a la reflexión. Este lector moderno, que elije, procesa, dialoga con el texto y lo interpela; ese lector que todos proponen en las instituciones de educación para formar ciudadanos libres e ilustrados, ese lector es, en perspectiva histórica, un invento bastante reciente.

Algunos antecedentes a nivel internacional, dejan en evidencia trabajos realizados por docentes que han tenido gran acogida en sus alumnos. Un estudio realizado en Perú propuso estrategias de com- prensión lectora basadas en las TIC como alternativa para mejorar el proceso de lectura en primaria, donde se creó e implemento una plataforma virtual llamada LEO, la cual busca mejorar la comprensión lectora, en textos narrativos y vocabulario, se trabajó con el grado quinto de primaria, obteniendo como resultado, mejores puntajes en las pruebas de lectura, (Thorne, Morla, Uccelli, Nakano, Mauchi, Landeo, Vásquez, \& Huerta, 2011).

En Venezuela se desarrolló un proyecto de investigación que busco promover el uso de las TIC en la educación donde a través de los recursos tecnológicos de maestros y estudiantes se fomentó el uso adecuado de los mismos, donde a través de actividades académicas se implementó el uso de 20 recursos online en pro del uso adecuado de las TIC en la educación, (Altamar, 2010). En España se desarrolló un trabajo de investigación titulado: leer bien para escribir mejor, el cual utilizó la página web como recurso digital, con información sobre lectura y actividades grupales e individuales, proponiendo talleres adecuados para las edades de los estudiantes, buscando fortalecer las capacidades para adquirir hábitos lectores y luego desarrollar actividades escritas, potenciando la participación activa del estudiante mediante sus competencia, y que incidieron en los procesos de investigación.

En Colombia los avances tecnológicos han influenciado el ámbito educativo integrando las TIC al aula de clase. En putumayo implementaron un software educativo para contribuir en el afianzamiento de la lectoescritura en los estudiantes del grado segundo básica primaria, en un proyecto llamado "Me divierto y aprendo lectoescritura", donde los docentes debían buscar herramientas virtuales para implementarlas en el salón de clases para mejorar las capacidades comprensivas y analíticas, (Cruz, 2012). 
Las competencias lectoras y escritoras son fundamentales en la enseñanza desde básica primaria, puesto que estas propician el desarrollo de todas las áreas del conocimiento, en la actualidad los jóvenes presentan poco interés por desarrollar este proceso, por tal razón surge la necesidad de fortalecer las competencias lectoras a través de la investigación como estrategia pedagógica (IEP) en la Institución Educativa Departamental Fossy Marcos María.

El estudio surge por la importancia de generar espacios de apropiación social donde se presenten propuestas pedagógicas mediante la integración de la investigación en las aulas, innovando el proceso de educación a partir de la creación de escenarios de reflexión y actualización para los docentes, donde puedan proponer metodologías para impactar en variables comunes dadas por problemáticas y realidades escolares propias de las instituciones educativas, ratificando la existencia de diversas prácticas de aula que pueden ayudar a transformar la cultura ciudadana de del municipio, propiciando la participación ciudadana.

En la IED Fossy Marcos María, sede principal y sede las delicias se observa que en los grados de tercero a quinto en el nivel de básica primaria hay niños que no saben leer y escribir correctamente; entre algunos de los problemas más notorios se destacan: confusión de las letras y sonidos, dificultad en el dictado, lectura poco fluida y escritura lenta, silábica y falta de comprensión, conllevando problemas a nivel cognitivo y dificultades de aprendizaje como; afasias del lenguaje conocidas como dislalia, dislexia, disgrafia, las anteriores son patologías del aprendizaje que en su gran mayoría los docentes no cuentan con las competencias o conocimientos para manejar dichos casos y propiciar una educación inclusiva, desconociendo las teorías que sustenten el desarrollo de la lectura y escritura en los estudiantes.

En este sentido, los docentes luego de realizar un diagnóstico en el área de humanidades para medir fortalezas y debilidades en la lectura y la escritura de los estudiantes que cursan los grados de tercero a quinto arrojo que cuarenta y dos (42) niños de la institución no saben leer y escribir. Situación problema que motivó a desarrollar el presente estudio para fortalecer competencias lectoras con el objetivo de mejorar la fluidez, comprensión lectora y producción oral y escrita.

Por tal motivo, la investigación en curso busca fortalecer las competencias lectoras y escritoras a través de la IEP en la educación básica primaria. Beneficiando el desarrollo de habilidades lecto-escritoras, la escuela dinamiza estos procesos cuando el docente le permite al estudiante intercambiar ideas, expresar puntos de vista, informarse, expresar su pensamiento e imaginar mundos posibles a través de la lectura y escritura, lo que permite apoyarse en la IEP, generando actividades que contribuyan a mejorar dicho proceso.

\section{Competencias lectoras y escritoras a través de la IEP: referentes teóricos}

Las características subyacentes en una persona, se pueden definir como las competencias, las cuales están relacionadas estrechamente con la actuación y el desempeño de sus labores en el trabajo. Igualmente se conceptualiza como conjunto de conocimientos, destrezas y aptitudes necesarias para ejercer una profesión que caracteriza al individuo en el desempeño de sus labores, desde el ámbito cognoscitivo de su actuación.

La lectura y escritura son procesos que hacen parte de las competencias comunicativas estas se plantean como el 
conjunto de conocimientos, habilidades y destrezas que requiere el uso adecuado, correcto, coherente y estético tanto del código oral como del escrito, compuesto por habilidades como; comprensión, expresión, análisis, síntesis, identificación, comparación, creación y recreación de mensajes, centrándola en escuchar, hablar, leer y escribir de forma competente (Reyzábal, 2012).

En ese sentido, las competencias lectoras y escritoras son procesos necesarios para el desarrollo del pensamiento y razonamiento en los estudiantes, debido a que estos hacen parte de actividades diarias propias de la escuela, permitiendo las relaciones del individuo con la sociedad, puesto que propician las habilidades comunicativas, sin embargo, los docentes deben tener en cuenta las concepciones o elementos de los estilos de aprendizaje de cada individuo debido a que estos permean las ideas y el comportamiento, por lo cual se requiere un estudio de los mismos de manera que posibilite el desarrollo adecuado de dichos procesos, es decir, los docentes deben identificar cual es el canal de aprendizaje de cada estudiante para diseñar estrategias que permitan su desarrollo e interiorización (Botello, 2013).

Por su parte, la lectura es un proceso que debe ser estimulado desde la infancia por padres y profesores, desde la escuela los docentes deben emplear estrategias pedagógicas para crear un habito de lectura en sus estudiantes, formándolos en competencias lingüísticas para que posteriormente puedan aportar nuevo conocimiento, originando así múltiples transformaciones en la producción de saber. Si la lectura se estimula desde temprana edad, los individuos adquieren un interés por la misma y les permite desarrollar habilidades lingüísticas (González, 2013).
De tal manera, que la competencia o habilidad lectora es la capacidad de captar la coherencia de un texto, reconstruyendo su mensaje, de acuerdo con la situación y la función comunicativa subyacente, por lo cual es necesario que el individuo se entrene para la adquisición de destrezas que le permitan desarrollar el pensamiento crítico, razonamiento, capacidad de análisis y comprensión, siendo estos elementos cognitivos que favorecen la comunicación con los otros. En este sentido, juegan un papel muy importante los docentes, que, como líderes, deben propiciar herramientas que nutran los procesos de interacción entre los miembros de la comunidad educativa; esto con el propósito de enriquecer el quehacer diario dentro de la organización, lo cual permite a su vez el desarrollo de competencias que fortalezcan las diferentes áreas del conocimiento.

Según González (2013), la decodificación del significado del vocabulario son de mucha ayuda en el diccionario, donde por medio de las diferentes actividades se recomiendan para fianzar criterios en el aula de clases, en ese sentido, dichas propuesta son pertinente a cuestionarios adecuados a las diferentes fases de interacción texto-lector, la realización de resúmenes orales y escritos para detectar la capacidad de síntesis del lector, el hábito de redactar con esquemas previos para detectar la capacidad de amplificación y organización discursiva, el hábito de revisar la cohesión sintáctica, la corrección ortográfica y la adecuación léxica de los propios escritos. Y la promoción de coloquios significativos, a partir de la lectura, para que aprendan a expresar sus ideas, a mostrar respeto e interés por las ajenas, y afianzar habilidades de interacción socio-comunicativa. 
Por su parte la escritura, es una de las cuatro habilidades básicas desarrolladas por el hombre en su dimensión comunicativa, esta es valorada como una herramienta de la humanidad que permite la interacción entre individuos, épocas y visiones del mundo. La escritura propone una ruptura espacio-temporal de las relaciones entre emisor y receptor, estableciendo una distancia entre el habla y su contexto. Es decir, amplía las posibilidades de la palabra y su trascendencia, hecho que le otorga una importancia histórica y ha sido la base para la evolución de la sociedad humana (Botello, 2013).

Según Molina \& Lucero (2013), la escritura fue desarrollada por el hombre como un medio para expresar ideas y pensamientos, convirtiéndose en una forma de socialización compleja, debido a la abstracción de la misma. Actualmente existen diversos soportes digitales y tecnológicos que permiten llevar a cabo este proceso, facilitando y transformando la forma de impartir conocimientos en el aula de clase. Los métodos tradicionales plantean que para la enseñanza de lectura y escritura el estudiante debe alcanzar la habilidad de decodificar los elementos que conforman el texto escrito y después descifrar el significado o contenido. Sin embargo, la inclusión de estrategias pedagógicas basadas en la investigación, pueden fomentar el desarrollo de los mismos procesos, de una forma agradable a partir de un aprendizaje contextual.

En la actualidad es importante comprender que la formación del estudiante no puede concebirse de forma aislada, sino como que es necesario, construir saberes asociados a las experiencias significativas de los mismos, generando practicas curriculares orientadas al contexto, de esta manera los planes de estudios deben estar orientados a la construcción social, partiendo del hecho que la escuela es un espacio de interacción, mediante la formación de estudiantes con competencias generadas desde la interdisciplinariedad. Los procesos de lectura y escritura están contemplados dentro de las competencias comunicativas, estas como herramienta social posibilitan el intercambio de saber y conocimiento, pensamientos, sentimientos, posturas frente a situaciones cotidianas, recreando la realidad, que favorece la construcción de significados y el uso de formas del lenguaje ajustadas a las necesidades y condiciones culturales del contexto en el que el individuo se desenvuelve (Marín, Niebles, Sarmiento \& Valbuena, 2017; Franco, 2012)

La interpretación comunicativa y la comunicación practica del acto comunicativo; son procesos se dan en la interacción social, de manera simultánea y compleja; desde el trabajo pedagógico se hace énfasis en competencias necesarias para fortalecer los procesos de lectura y escritura, estos son: Competencia gramatical o sintáctica; que incluye reglas sintácticas, morfológicas, fonológicas y fonéticas que rigen la producción de los enunciados lingüísticos. La competencia textual; que se refiere a los mecanismos que garantizan coherencia y cohesión a los enunciados de los textos. Competencia semántica; que es la capacidad de reconocer y usar los significados de manera pertinente según las exigencias del contexto comunicativo (Arce, 2005).

Por otro lado, la competencia pragmática o sociocultural, es entendida como el reconocimiento de las intencionalidades del contexto como el componente ideológico y político que está detrás de los enunciados. La competencia 
enciclopédica, referida a la capacidad de poner en juego, en los actos de significación y comunicación, los saberes con los que cuentan los hablantes y que son construidos en el ámbito de la cultura académica o socio-cultural, local y familiar. Finalmente, la competencia literaria, implica la capacidad de poner en juego, en los procesos de escritura, un saber literario surgido de la experiencia de lectura, análisis de las obras y del conocimiento directo de un número significativo de éstas (Arce, 2005)

En este punto es necesario definir las estrategias pedagógicas, estas son procesos, que implican la planificación y uso de recursos metodológicos para impartir conocimientos en el aula, se trata de toda una serie de conceptos y procesos que se entraman para garantizar la eficacia de la organización. Estos conceptos se basan en fuerzas y las debilidades de la misma, los cambios del ambiente y los movimientos de los competidores. Chiavenato (2009), manifiesta que estrategia es la movilización de todos los recursos de la institución en el ámbito global para conseguir objetivos planteados. También se refiere como el conjunto de objetivos y políticas, con la finalidad de guiar y orientar la organización a un comportamiento que garantice el éxito.

En este sentido, las estrategias didácticas utilizadas en el subsistema de educación básica primaria deben ser diseñadas de tal manera que estimulen a los participantes a observar, analizar, opinar, formular hipótesis, buscar soluciones y descubrir el conocimiento por sí mismos. En consideración a esto, se hace necesario mencionar a Martínez, (2014) el cual expone que "deben existir nuevas estrategias pedagógicas, con las cuales el docente pueda ser un verdadero mediador al ofrecer lo que el estudiante quiere en un momento oportuno y despertar en ellos la capacidad de crear de manera productiva”.

La investigación como estrategia pedagógica ejerce un impacto positivo al integrarla al aula de clases, brindándole a la comunidad educativa la posibilidad de construir nuevo conocimiento apropiando herramientas científicas, que respondan a una necesidad o problemática social, proyectando al estudiante hacia un aprendizaje significativo, (Ausubel, 2002). En la implementación de la IEP los contenidos educativos se convierten en problemáticas de posible abordaje y el currículo pasa a estructurarse a partir del surgimiento de una serie de preguntas educativas expuestas por los estudiantes, el avance y desarrollo de los mismos se manifiesta a través del lenguaje, razonamiento y conocimiento que ellos demuestren; ese descubrimiento generalmente no se hace de manera autónoma, es guiado por el docente que se encarga de planificar las estrategias, los ejercicios y los procedimientos para el fin búsqueda (Mejía \& Manjarrés, 2010).

En efecto, al considerar lo antes expuesto, las competencias lectoras y escritoras a través de la IEP, de un manejo a los referentes teóricos operacional-izados, reafirma que la existencia de conocimientos y reflexiones en la acción, lo que permite integrar en las actuaciones explícitas e implícitas lo cognitivo, emocional, la teoría y práctica. Reconociendo que tanto quien enseña como quien aprende dentro de una comunidad trabajan para generar conocimiento local, prever su práctica y teorizar sobre ella, interpretando las conclusiones de otros, todo lo anterior es posible al integrar la investigación al aula (ver tabla 1). 
TABLA 1

Operacionalización de referentes teóricos de las competencias lectoras y escritoras a través de la IEP

\begin{tabular}{|c|c|}
\hline $\begin{array}{c}\text { Referentes } \\
\text { teóricos }\end{array}$ & Competencias lectoras y escritoras a través de la IEP \\
\hline Botello (2013). & $\begin{array}{l}\text { Procesos necesarios para el desarrollo del pensamiento y razonamiento en los estudiantes, } \\
\text { permitiendo las relaciones del individuo con la sociedad, propician habilidades } \\
\text { comunicativas, considerando las concepciones o elementos de los estilos de aprendizaje } \\
\text { de cada individuo, permeando en las ideas y comportamiento del alumno. }\end{array}$ \\
\hline $\begin{array}{l}\text { González } \\
\text { (2013). }\end{array}$ & $\begin{array}{l}\text { Proceso que debe ser estimulado desde la infancia por padres y profesores, empleando } \\
\text { estrategias pedagógicas para crear un hábito de lectura en sus estudiantes, formándolos } \\
\text { en competencias lingüísticas para que posteriormente puedan aportar nuevo conocimiento, } \\
\text { originando así múltiples transformaciones en la producción de saber y gestión del } \\
\text { conocimiento. }\end{array}$ \\
\hline $\begin{array}{l}\text { Molina \& } \\
\text { Lucero (2013). }\end{array}$ & $\begin{array}{l}\text { Un medio para expresar ideas y pensamientos, convirtiéndose en una forma de } \\
\text { socialización compleja, debido a la abstracción de la misma. Actualmente existen diversos } \\
\text { soportes digitales y tecnológicos que permiten llevar a cabo este proceso, facilitando } \\
\text { y transformando la forma de impartir conocimientos en el aula de clase. Los métodos } \\
\text { tradicionales plantean que para la enseñanza de lectura y escritura el estudiante debe } \\
\text { alcanzar la habilidad de decodificar los elementos que conforman el texto escrito y después } \\
\text { descifrar el significado o contenido. La inclusión de estrategias pedagógicas basadas en } \\
\text { la investigación, pueden fomentar el desarrollo de los mismos procesos, de una forma } \\
\text { agradable a partir de un aprendizaje contextual. }\end{array}$ \\
\hline $\begin{array}{l}\text { Reyzábal } \\
(2012) .\end{array}$ & $\begin{array}{l}\text { Procesos que hacen parte de las competencias comunicativas, se plantean como el } \\
\text { conjunto de conocimientos, habilidades y destrezas que requiere el uso adecuado, correcto, } \\
\text { coherente y estético tanto del código oral como del escrito, compuesto por habilidades } \\
\text { como; comprensión, expresión, análisis, síntesis, identificación, comparación, creación } \\
\text { y recreación de mensajes, centrándola en escuchar, hablar, leer y escribir de forma } \\
\text { competente. }\end{array}$ \\
\hline $\begin{array}{l}\text { Mejía \& } \\
\text { Manjarrés } \\
(2010) .\end{array}$ & $\begin{array}{l}\text { Convierte problemáticas de posible abordaje, a partir del surgimiento de una serie de } \\
\text { preguntas educativas expuestas por los estudiantes, el avance y desarrollo de los mismos } \\
\text { se manifiesta a través del lenguaje, razonamiento y conocimiento que ellos demuestren; ese } \\
\text { descubrimiento generalmente no se hace de manera autónoma, es guiado por el docente } \\
\text { que se encarga de planificar las estrategias, los ejercicios y procedimientos para el fin } \\
\text { búsqueda, el aprendizaje. }\end{array}$ \\
\hline
\end{tabular}

Fuente: elaboración propia.

\section{Metodología}

\section{Diseño}

El presente estudio se abordó bajo los lineamientos de la IEP, con un diseño basado en las trayectorias de indagación, utilizando técnicas propias de la investigación cualitativa necesaria para la recolección de información. El enfoque epistemológico es de tipo cualitativo utilizando herramientas para la recolección de datos como observación participante y diario de campo, el estudio se desarrolló a la luz del diseño de estrategias pedagógicas que mediante la IEP que motiven a los estudiantes fortalecer competencias y mejorar la comprensión lectora.

\section{Participantes}

Como unidad de análisis se seleccionaron cuarenta y dos (42) estudiantes de tercero a quinto de las sedes delicia y principal de la Institución Fossy Marcos María con edades comprendidas entre los 8 y los 10 años. Cabe destacar que los participantes diligenciaron debidamente el consentimiento informado. 


\section{Técnicas e instrumentos}

En efecto, la observación participante es un tipo de método de recolección de datos utilizado típicamente en la investigación cualitativa. Es una metodología ampliamente utilizada en muchas disciplinas, particularmente la antropología y la etnología, aunque también en sociología, estudios de comunicación, geografía humana y psicología social. Mientras que el diario de campo es un instrumento utilizado por los investigadores para registrar aquellos hechos que son susceptibles de ser interpretados. Es una herramienta que permite sistematizar las experiencias para luego analizar los resultados.

Respecto al proceso formativo, en la observación participante y diario de campo, se evidencia en el estudiante el desarrollo del sentido crítico, propiciador y potenciador de producción intelectual. Toma conciencia de las condiciones particulares y asumir una postura de aceptación y búsqueda de superación de las limitaciones, preconceptos y pre-juicios, siendo un avance formativo, difícilmente evidenciable en otro tipo de fuentes o estrategias de información para la evaluación del estudiante.

\section{Procedimiento}

A partir del recorrido de las trayectorias de indagación se diseñaron tres fases, la primera diagnostica, donde se observó a los estudiantes dentro del aula de clases para establecer las condiciones iniciales de los sujetos. En la siguiente fase se realizará una entrevista estructurada a los docentes del área de lenguaje, una para identificar que estrategias están usando en el mejoramiento de la comprensión lectora y cuál es su percepción respecto a la efectividad de las mismas. En una fase posterior, con base al diagnóstico levantado, se diseñaron las estrategias que motivan al estudiante a usar la lectura como herramienta fundamental de superación y medio para alcanzar un pensamiento investigativo. A continuación, se presentan los diferentes momentos.

Momento (1): selección y análisis de la población sujeto de estudio.

Momento (2): integración de la temática mediante la IEP al aula de clases, se integraron grupos estudiantiles para seleccionar problemáticas relacionadas con la violencia sexual. Los estudiantes se plantearon preguntas y con el acompañamiento del docente realizaron el proceso de indagación mediante herramientas virtuales y entrevistas a la comunidad.

Momento (3): socialización de los resultados de las indagaciones de estudiantes en una feria institucional dirigida por los docentes a cargo.

Momento (4): análisis de los resultados de la implementación de la estrategia a través de la observación y diario de campo.

Momento (5): propagación y divulgación de los resultados.

\section{Resultados}

Se observó que en los grados de tercero a quinto en el nivel de básica primaria hay niños que no saben leer y escribir correctamente; entre algunos de los problemas más notorios se destacan: confusión de las letras y sonidos, dificultad en el dictado, lectura poco fluida y escritura lenta, silábica y falta de comprensión, conllevando problemas a nivel cognitivo y dificultades de aprendizaje como; afasias del lenguaje conocidas como dislalia, dislexia, digrafía, las anteriores son patologías del aprendizaje que en su gran mayoría los docentes no cuentan con las competencias o conocimientos para manejar dichos casos y propiciar una educación inclusiva, desconociendo las teorías que sustenten el desarrollo de la lectura y escritura en los estudiantes.

Los docentes luego de realizar un diagnóstico en el área de humanidades para medir fortalezas y debilidades en la lectura y la escritura de los estudiantes que cursan los grados de tercero a quinto arrojo que cuarenta y dos (42) niños de la institución 
no saben leer y escribir. Situación problema que motivó a desarrollar el presente estudio para fortalecer competencias lectoras con el objetivo de mejorar la fluidez, comprensión lectora y producción oral y escrita. Al mismo tiempo, los resultados para fortalecer las competencias lectoras y escritoras a través de la IEP en la educación básica primaria, se orientan por la sistematización de indicadores, considerando:

a. En efecto, la lecturas y escritura: en la institución educativa debe contar con una colección textos variada, moderna y bien seleccionada de lecturas; organizada y centralizada como una medida de consulta pertinente al contexto.

b. Dedicación por la por la lectura y escritura: se lee para que guste, sin la obligatoriedad de trabajos posteriores que supongan una carga académica, por el contrario, se busca es que el lector se involucre con un sentido de entrega por la lectura

c. Tiempo y más dedicación a la lectura y escritura: facilitar espacios propicios y programar tiempos para la lectura en el aula, biblioteca escolar, en rincones adaptados para ello, en los patios, dinamizar esos espacios y esos tiempos con ayuda del alumnado.

d. Leer en voz alta: el estudiante, se recupera el placer de la lectura por la lectura en voz alta y fomenta el intercambio de lecturas.

e. La lectura y escritura es de las personas, no de las asignaturas: todo el profesorado se implica en potenciar el hábito lector en el centro, no corresponde a una asignatura o a un área concreto monopolizar el gusto por la lectura, sino que es la comunidad educativa la que lee y anima a leer.

f. A los efectos de este, la diversidad de lecturas para la escritura: el hábito lector no sólo se refiere al gusto por la literatura o por alguno de sus géneros, sino que también incluye los libros informativos, cómics, diarios y revistas.

\section{Conclusiones}

Las competencias lectoras-escritoras son procesos necesarios para desarrollar el pensamiento y razonamiento en los estudiantes, al hacer parte de actividades diarias de la escuela, permitiendo relacionar al individuo y la sociedad, al propiciar las habilidades comunicativas, sin embargo, los docentes deben tener en cuenta las concepciones o elementos de los estilos de aprendizaje de cada individuo debido a que estos permean las ideas y el comportamiento, por lo cual se requiere un estudio de los mismos de manera que posibilite el desarrollo adecuado de dichos procesos, es decir, los docentes deben identificar cual es el canal de aprendizaje de cada estudiante para diseñar estrategias que permitan su desarrollo e interiorización (Botello, 2013).

En este aspecto, se recomienda resignificar la labor docente y adoptar una posición como lectores de las hipótesis de los niños y sumar una actitud abierta al cambio, y así seguramente se formarán lectores y escritores desde el primer día de clase. Al fortalecer las competencias lectoras y escritoras a través de la IEP en la educación básica primaria, se busca cuestionar las concepciones de la lecto-escritura, al ser posible que el conocimiento al respecto no sea claro y coherente al obstaculizar de alguna forma los intereses de los niños en la escuela, al pretender que aprendan a leer y escribir en el menor tiempo posible para cumplir con exigencias del mercado, sin tener en cuenta que ellos son escritores-lectores en potencia y necesitan es un maestro orientador, mediador y facilitador del aprendizaje adaptado a sus necesidades.

Alcanzar la competencia lectora y escritura, supone un aprendizaje amplio, multidimensional, que requiere la movilización de capacidades cognitivas, afectivas y de inserción social. Es un esfuerzo para los, docentes y discentes, pero es un esfuerzo que vale la pena, pues de habla de uno de los aprendizajes más funcionales y capacitadores que puede hacer una persona; de una llave que abre múltiples posibilidades de desarrollo y crecimiento académico, profesional y personal. 


\section{Referencias}

Acosta, S. y García, M. (2012). Estrategias de enseñanza utilizadas por los docentes de biología en las universidades públicas. Rev. Omnia, 18(2). 67-82.

Altamar, M. (2010). Estrategias que promuevan el uso de las TIC. Venezuela: Liceo Bolivariano Evelia Ávila De Pimentel.

Arce, G. (2005). Las competencias comunicativas en el contexto pedagógico. Revista Ingenio Libre. 4. 1-12.

Ausubel, D. (2002) Adquisición y retención del conocimiento. Madrid: Ed. Paidós.

Botello, S. (2013). La escritura como proceso $y$ objeto de enseñanza. [Tesis de maestría]. Universidad del Tolima, Ibagué.

Carr, N. (2011). Superficiales ¿Qué está haciendo internet con nuestras mentes? Madrid: Taurus.

Chiavenato, I. y Villamizar, G. (2009). Gestión del talento humano. México, D.F.: McGraw-Hill.

Cochran, M. y Litle, S. (2006). Más allá de la certidumbre adoptar una actitud indagadora sobre la práctica. Barcelona: Octaedro.

Cruz, L. (2012). Proyecto de aula en TIC, me divierto y aprendo lectoescritura. Pasto: Universidad de Nariño.

Franco, M. (2012). Estrategias de enseñanza para la promoción de la comprensión lectora desde el aprendizaje significativo. Cultura, Educación, Sociedad, 3(1), 175186.

González, D. (2013). Importancia de los para textos en la lectura e Interpretación de Textos Literarios. Rev. Multiciencias. 180-189.

Jiménez, J. y Salas, M. (2017). Aplicación de modelos econométricos para estimar la aceptabilidad de una tasa por congestión vehicular. INGE CUC, 13(2), 60-78. https:// doi.org/10.17981/ingecuc.13.2.2017.08
Marín, F., Niebles, M., Sarmiento, M. y Valvuena, S. (2017). Mediación de las tecnologías de la información en la comprensión lectora para la resolución de problemas aritméticos de enunciado verbal. Revista Espacios, 38(20).

Martínez, M. (2014). Los estilos de aprendizaje de los estudiantes y las estrategias didácticas de enseñanza de los maestros de matemática del noveno año de educación general básica de la ciudad de Cuenca. [Master's thesis].

Mariño, G. (2010). El diálogo en la educación de jóvenes y adultos. Dos propuestas pedagógicas para implementarlo. En: El taller dialógico / la recuperación de experiencias laborales. Bogotá, D.C.: OEI.

Mejía, M. y Manjarrés, M. (2010). Las pedagogías fundadas en la investigación. Búsquedas en la reconfiguración de la educación. Revista Internacional Magisterio 42. 16-26.

Molina, P. y Lucero, M. (2013). Propuesta pedagógica para mejorar la competencia interpretativa. [Doctoral dissertation]. Universidad de la Sabana, Bogotá, D.C.

Ortega, P., Peñuela, D. y López, D. (2009). Sujetos y prácticas de la pedagogía crítica. Bogotá, D.C.: Ediciones El Búho.

Pozo, J. y Gómez, M. (2006). Aprender y enseñar ciencia. Madrid: Morata.

Reyzábal, M. (2012). Las Competencias Comunicativas y Lingüísticas, Clave Para La Calidad Educativa. Reice. Revista Iberoamericana sobre Calidad, Eficacia y Cambio en Educación, 10(4), 63-77.

Thorne, C, Morla, K., Uccelli, P., Nakano, T., Mauchi, B., Landeo, L., Vásquez, A. y Huerta, R. (2011). Estrategias de comprensión de lecturas mediadas por TIC. Una alternativa para mejorar las capacidades lectoras en primaria. [Tesis]. Pontificia Universidad Católica del Perú, Lima. 